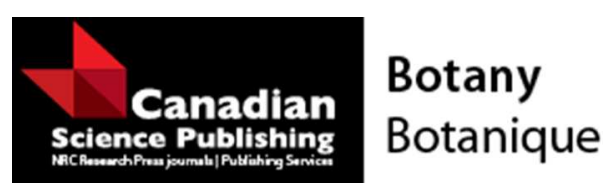

\title{
An efficient temporary Immersion system for micropropagation of hybrid hazelnut
}

\begin{tabular}{|r|l|}
\hline Journal: & Botany \\
\hline Manuscript ID & cjb-2015-0111.R1 \\
\hline Manuscript Type: & Methods \\
\hline Date Submitted by the Author: & 23-Sep-2015 \\
\hline Complete List of Authors: & $\begin{array}{l}\text { Latawa, Jyoti; University of Guelph, Plant Agriculture } \\
\text { Shukla, Mukund; University of Guelph, Plant Agriculture } \\
\text { Saxena, Praveen; University of Guelph, Plant Agriculture }\end{array}$ \\
\hline Keyword: & $\begin{array}{l}\text { Temporary Immersion System, FeEDDHA, Iron source, Corylus, } \\
\text { micropropagation }\end{array}$ \\
\hline \multicolumn{2}{|c}{} \\
\hline
\end{tabular}

\section{SCHOLARONE ${ }^{\text {I" }}$}

Manuscripts 
An efficient temporary immersion system for micropropagation of hybrid hazelnut

\author{
Jyoti Latawa, Mukund R. Shukla and Praveen K. Saxena*
}

Department of Plant Agriculture, Gosling Research Institute for Plant Preservation, University of Guelph, Guelph, ON, Canada

Email addresses of authors: Jyoti <jyoti@uoguelph.ca>, Mukund R. Shukla $<$ mshukla@uoguelph.ca>,Praveen K. Saxena<psaxena@uoguelph.ca>

*Corresponding Author: Praveen Saxena (e-mail: psaxena@uoguelph.ca).

Phone No.: 5198244120 Ext 52495. 


\begin{abstract}
An efficient protocol for micropropagation of hybrid hazelnut (Corylus) was developed using Temporary Immersion System (TIS) for multiplication of shoots in the liquid medium. Growth and multiplication of shoots as well as the length and number of nodes in developing shoots were significantly improved by combining two forms of chelated iron, ethylenediamine-N,N'-di-(ortho-hydroxyphenyl) acetic acid (FeEDDHA) and $\mathrm{FeSO}_{4} /$ EDTA (FeEDTA). The use of TIS with optimized concentrations of FeEDTA and FeEDDHA significantly increased the number of shoots, shoot height, leaf area and chlorophyll content in all of the four cultivars tested compared to semi-solid medium. Proliferated shoots developed roots on a semi-solid medium containing $2.5 \mu \mathrm{M}$ IBA and rooted plantlets acclimatized in greenhouse conditions with $80 \%$ survival.
\end{abstract}

Keywords: Corylus, FeEDDHA, iron source, Temporary Immersion System, bioreactor, micropropagation 


\section{Introduction}

Hazelnut (Corylus avellana L.) is the fourth most economically important nut crop after cashew, almond and walnut and global demand for hazelnut based food products is increasing consistently. Annual Canadian import of hazelnut exceeds 16 million pounds indicating a great potential of this crop in agricultural and food industries in Canada. Micropropagation offers excellent opportunity for large-scale production of plants since conventional methods are slow, time consuming and inefficient (Garrison et al. 2013). Minor nutrients are critical for an improved growth of Corylus avellana shoot cultures (Hand et al. 2014; Hand and Reed 2014). However, hazelnut shoot multiplication in vitro is very sensitive to the concentration and type of iron source (Yu and Read 1995; Garrison et al. 2013). Two commonly used ironsources in plant tissue culture media are ethylenediamine tetra-acetic acid (Fe(III)-EDTA) (FeEDTA) and Fe(III)-chelate of ethylenediamine-N,N'-di-(ortho-hydroxyphenyl) acetic acid (Fe(III)-o,o-EDDHA) ( FeEDDHA). Several studies with these two chelating agents have shown enhanced growth and proliferation of hazelnut on the semi-solid medium supplemented with FeEDDHA (Bassil et al. 1992; Yu and Read 1995; Nas and Read 2001, 2004; Garrison et al. 2013). In this communication we demonstrate the advantage of combining two different sources of iron in developing a simple and highly efficient temporary immersion culture system for rapid shoot proliferation of hazelnut.

\section{Materials and Methods}

\subsection{Plant material}

The hazelnut hybrid C. avellana L. x C. americana M cv. Geneva (Grimo Nut Nursery, Niagara-on-theLake, ON) was used for standardizing the protocol while other hybrid hazelnut (Corylus sp.) cultivars viz., C-16 (Martin Hodgson, London, ON, Canada), Epsilon and Jefferson were used in subsequent experiments to compare the effects of genotypic variation. In vitro shoot cultures of Geneva and C-16 were originally derived from greenhouse grown plants, while Epsilon and Jefferson were obtained from North American Plant Inc. (Oregon, USA). Nodes with single shoot buds were collected from March to September from two 
year old plants grown in the greenhouse. Buds were washed in running tap water for 60 min and sterilized by dipping in $70 \%(\mathrm{v} / \mathrm{v})$ ethanol for one min followed by immersion in a $20 \%(\mathrm{v} / \mathrm{v})$ Clorox ${ }^{\circledR}$ solution $(5.4 \%$ sodium hypochlorite) for $20 \mathrm{~min}$ with intermittent agitation and three rinses in sterile distilled water (4 min). Individual explants were cultured in test tubes each containing $8 \mathrm{ml}$ of liquid or semi-solid basal DKW (Driver and Kaniyuki 1984) medium supplemented with 3\% (w/v) sucrose, 2 ml/1 Plant Preservative Mixture $\left(\mathrm{PPM}^{\mathrm{TM}}\right.$, PhytoTechnology Shawnee Mission, KS), and 2.2 g/l phytagel (Sigma-Aldrich, USA) as the solidifying agent. After four weeks, developed shoots were excised and maintained in magenta boxes (Magenta Corporation, Chicago, IL) containing $50 \mathrm{ml}$ of semi-solid basal DKW medium supplemented with BA $(17.6 \mu \mathrm{M}), \mathrm{GA}_{3}(0.29 \mu \mathrm{M})$ and IBA $(0.14 \mu \mathrm{M})$ according to Garrison et al. (2013).

\subsection{Culture growth and maintenance}

Temporary immersion system (TIS) involves a temporary immersion of the explant in the liquid medium for specific interval of time, thereby providing the medium at one interval and oxygenation during the other. The Liquid Lab Rocker ${ }^{\mathrm{TM}}$ (Southern Sun BioSystems, Inc., Hodges, SC) is a simple Tilting-Rocker machine, which tilts the Liquid $\mathrm{Lab}^{\mathrm{TM}}$ vessels at an angle in both the directions thereby intermittently immersing the explant into the medium for a specific period of time. Explants (5-6 nodal segments) excised from 8 week old cultures grown on semi-solid medium were transferred to Liquid Lab ${ }^{\mathrm{TM}}$ Rocker temporary immersion bioreactor (TIS) vessels containing $50 \mathrm{ml}$ of liquid DKW medium of the same composition as above (Fig. 1a). Southern Sun Liquid Lab ${ }^{\mathrm{TM}}$ vessels (10.5"x4.0”x4.0") were maintained on a Liquid Lab ${ }^{\mathrm{TM}}$ Rocker bioreactor system with rocker speed set to $30 \mathrm{sec} / \mathrm{cycle}$ thereby immersing the explants with an interval of $25 \mathrm{sec}$ between each cycle. The medium was replaced with the fresh $50 \mathrm{ml}$ medium after every three weeks. Cultures grown on semi-solid media in Magenta ${ }^{\circledR}$ GA7 boxes (Phytotechnology, KS, USA) were also sub-cultured after every three weeks. All cultures were maintained at $22^{\circ} \mathrm{C}$ with $16 \mathrm{~h}$ photoperiod $\left(40 \mu \mathrm{mol} \mathrm{m} \mathrm{s}^{-2}\right)$. 


\subsection{Effect of iron supplements on growth}

Effects of iron sources on the growth of Geneva were tested with a commercial DKW (CDKW) and a modified DKW (MDKW) media. The CDKW basal salt (PhytoTechnology Shawnee Mission, KS) contained $\mathrm{FeSO}_{4}$ and $\mathrm{Na}_{2}$ EDTA each at $121.58 \mu \mathrm{M}$ whereas the MDKW medium was prepared with CDKW composition devoid of $\mathrm{FeSO}_{4}$ and $\mathrm{Na}_{2}$ EDTA. Explants derived from established shoot cultures were grown in TIS using a liquid commercial DKW culture medium (CDKW) containing FeEDTA (121.58 $\mu \mathrm{M}$ each of $\mathrm{FeSO}_{4}$ and $\mathrm{Na}_{2} \mathrm{EDTA}$ ) and a modified medium lacking $\mathrm{FeSO}_{4}$ and $\mathrm{Na}_{2}$ EDTA (MCKW). FeEDDHA was added as the sole iron source to MCKW and as an additional source of chelated iron to CDKW media. Both media were supplemented with BA $(17.6 \mu \mathrm{M}), \mathrm{GA}_{3}(0.29 \mu \mathrm{M})$, IBA $(0.014 \mu \mathrm{M})$ (Garrison et al. 2013) and glucose (3\%). Explants with 5-6 nodes were cultured in TIS vessels each containing $50 \mathrm{ml}$ of autoclaved CDKW or MDKW medium containing FeEDDHA (PhytoTechnology, Shawnee Mission, KS) at $0,230,345,460$ and $575 \mu \mathrm{M}$. Observations on number of shoots/explant, average shoot height and average number of nodes per shoot were recorded after six weeks.

\subsubsection{Iron detection and quantification}

Iron uptake was investigated using Perl's Prussian blue/Diaminobenzidine (PPB/DAB) as described by Meguro et al. (2007). Briefly, leaves were vacuum infiltrated for $10 \mathrm{~min}$ in a freshly prepared solution containing $4 \%(\mathrm{v} / \mathrm{v}) \mathrm{HCl}(37 \%)$ and $4 \%(\mathrm{w} / \mathrm{v})$ potassium-ferrocyanide followed by incubation for $30 \mathrm{~min}$ at room temperature. Potassium-ferrocyanide reacts with ferric ions in the leaf tissue to produce a blue color referred to as Perl's Prussian blue (PPB). Leaves were washed with distilled water and incubated in $0.01 \mathrm{M}$ $\mathrm{NaN}_{3}$ and $0.3 \%(\mathrm{v} / \mathrm{v}) \mathrm{H}_{2} \mathrm{O}_{2}$ in methanol for 35 min until discoloration of the leaves occurred. Leaves were incubated in $100 \mathrm{mM}$ phosphate buffer ( $\mathrm{pH} 7.4$ ) containing $0.025 \%$ (w/v) DAB, $0.005 \% \mathrm{H}_{2} \mathrm{O}_{2}$, and $0.005 \%$ (w/v) $\mathrm{CoCl}_{2}$ for $5-6$ min to further intensify the blue color by oxidative polymerization with PPB which appeared as reddish brown spots. Finally, leaves were washed three times with $100 \mathrm{mM}$ phosphate buffer (pH 7.4) and observed under bright field (Axiovert200, Carl Zeiss Canada Ltd., Toronto, ON, Canada). Three leaves from each replicate of the treatments were collected randomly and stained as described above. 
ICP-AES (inductively coupled plasma atomic emission spectroscopy) was used to measure the iron content of plantlets grown in the medium with different iron types and concentrations according to Hirata et al. (1986). Plantlets from three individual replicates for each concentration of iron supplement were pooled and dried in oven for $24 \mathrm{~h}$ at $80^{\circ} \mathrm{C}$. Samples were homogenized and analyzed through ICP-AES at Lab Services Division (University of Guelph, Guelph, ON).

\subsection{Comparison of TIS and semi-solid system for growth of various hazelnut cultivars}

Growth performance of four hybrid hazelnut cultivars viz. Geneva, C-16, Epsilon and Jefferson were compared using TIS and semi-solid culture systems. Liquid medium for TIS was prepared using DKW basal salts with BA $(17.6 \mu \mathrm{M}), \mathrm{GA}_{3}(0.29 \mu \mathrm{M})$, IBA $(0.014 \mu \mathrm{M})$ and $460 \mu \mathrm{M}$ FeEDDHA. In semi-solid culture medium, phytagel was added as the solidifying agent at a concentration of $2.2 \mathrm{~g} / \mathrm{l}$ (pH 5.7). Nodal explants with 3-4 nodes were transferred to the magenta boxes and TIS vessels containing $50 \mathrm{ml}$ of autoclaved semi-solid and liquid medium, respectively. Observations were taken for the number of shoots produced per explant, shoot height, number of nodes in new shoots produced and chlorophyll content after 8 weeks of culturing. Multiplication rate was calculated by multiplying the number of shoots by the number of nodes. Leaf area was measured using ImageJ-win 64 software (Glozer 2008).

\subsubsection{Chlorophyll estimation}

Approximately 100-150 mg of fresh leaf tissue from each replicate (total four replicates) of a treatment were collected, weighed and cut into small pieces. Chlorophyll was extracted by incubating the chopped leaves in $8 \mathrm{ml}$ of $95 \%$ of ethanol for $48 \mathrm{~h}$ at $4^{\circ} \mathrm{C}$ in the dark. Absorbance of the supernatant was measured on a Beckmann Coulter DU-800 spectrophotometer at 664 and 648 nm against 95\% ethanol as blank. Total chlorophyll content i.e. Chlorophyll $\mathrm{a}$ and $\mathrm{b}$ was calculated using following formula according to Lichtenthaler et al. (2001).

$$
\mathrm{C}_{\mathrm{a}}(\mu \mathrm{g} / \mathrm{ml})=13.36 \mathrm{~A}_{664.1}-5.19 \mathrm{~A}_{648.6} \text { and } \mathrm{C}_{\mathrm{b}}(\mu \mathrm{g} / \mathrm{ml})=27.43 \mathrm{~A}_{648.6}-8.12 \mathrm{~A}_{664.1}
$$


where $C_{a}$ is chlorophyll $\mathrm{a} ; \mathrm{C}_{\mathrm{b}}$ is chlorophyll $\mathrm{b} ; \mathrm{A}_{664.1}$ is absorbance at wavelength $664.1 \mathrm{~nm} ; \mathrm{A}_{648.6}$ absorbance at wavelength $648.6 \mathrm{~nm}$.

\subsection{Effect of IBA on root induction}

Rooting was assessed with DKW basal medium containing $2 \%$ sucrose and IBA at $0,0.5,2.5,5$ and $10 \mu \mathrm{M}$ in the presence or absence of FeEDDHA $(460 \mu \mathrm{M})$. Each TIS vessel and culture tube was dispensed with $50 \mathrm{ml}$ and $8 \mathrm{ml}$ aliquotes of medium, respectively and $50 \mathrm{ml}$ of phytagel $(2.2 \mathrm{~g} / \mathrm{l})$ solidified medium was poured into each magenta box. For each concentration of IBA tested, four replicates were used each consisting of five magenta boxes for semi-solid medium and five test tubes for liquid medium containing a shoot with 7-8 nodes. In case of TIS, four replicates each containing five shoots with 7-8 nodes were used for each concentration of IBA.

In vitro rooted plantlets were removed from the culture media, washed gently with tap water, and transferred to 18-cell trays containing Professional growing mix 4 (Sun Gro Horticulture, Vancouver, BC). All trays were placed in a mist bed for two weeks before transfer to greenhouse (average temp $25 \pm 2{ }^{\circ} \mathrm{C}$ with a $16 \mathrm{~h}$ photoperiod). A total of 72 plantlets were transplanted into $4 \times 18$-celled trays to provide four replicates. Survival rate of the plantlets was recorded 4 weeks after transfer to the greenhouse.

\section{Statistical analysis:}

All experiments were conducted using a completely randomized design and analysis of variance was done using JMP Pro 10. Effect of different concentrations of two iron sources was analysed using one-way ANOVA and the interaction effects between the cultivars and physical state of medium were evaluated using factorial designs $(2 \times 4)$. All experiments were repeated at least twice. Means were compared using Tukey's test $(\mathrm{P}<0.05)$. The data are presented as means \pm standard error of means and same letters in figures indicate no significant difference at a p-value of 0.05 . 


\section{Results}

Initial experiments to establish culture protocol showed that the buds of hybrid hazelnut (C. avellana $\mathrm{L} . \mathrm{x}$ C. Americana M cv. Geneva) collected mid-May from greenhouse grown plants developed minimal contamination (data not shown). Direct culture of buds in liquid medium was unsuccessful as the explants underwent necrosis within 2 weeks of culture while a maximum of $30 \%$ axillary bud initiation was observed in the semi-solid medium (Figs. $1 \mathrm{~b}$ and c). Therefore, nodal explants were grown on a semi-solid medium to produce healthy cultures (Fig. 1d), which provided plant material for further experiments.

Explants failed to grow in the absence of an iron source whereas, minimal shoot development was observed with iron as FeEDTA. The addition of $230 \mu \mathrm{M}$ FeEDDHA to the medium as the sole source of iron promoted shoot development but the response gradually declined with increased FeEDDHA (Fig. 2a). However, this decline was absent in explants cultured with combined sources of iron (CDKW with FeEDDHA), where maximum shoot development was observed at $460 \mu \mathrm{M}$ FeEDDHA and $121 \mu \mathrm{M}$ FeEDTA. Addition of FeEDDHA, alone or in combination with FeEDTA, resulted in increased shoot height and number of nodes (Figs. 2b-c) up to $460 \mu \mathrm{M}$, but was inhibitory at $575 \mu \mathrm{M}$.

The PPB/DAB detection method confirmed variation in iron content of leaves from plants grown in different concentrations and forms of iron (Fig. 3a-c). Leaves from plants grown on CDKW medium with $121.58 \mu \mathrm{M}$ FeEDTA showed minimal reddish-brown spots that were more prominent near veins, indicating a low Fe content with localized distribution in the tissue (Fig. 3a). A more even distribution of reddishbrown spots was observed in leaves from plants grown in media with up to $460 \mu \mathrm{M}$ FeEDDHA alone or in combination with FeEDTA (Fig. 3b-c). This pattern was maintained up to $575 \mu \mathrm{M}$ of iron given in combination of both the iron sources. However, when iron was provided with FeEDDHA alone, it formed larger spots, suggesting accumulation and compartmentalization of iron. Iron accumulation was significantly greater in plants provided with a combination of FeEDDHA and FeEDTA (FeEDDHA added to CDKW) medium as compared to FeEDDHA alone (MDKW medium) up to the $460 \mu \mathrm{M}$ level (Fig. 3d). 
However, further increase to $575 \mu \mathrm{M}$ in FeEDDHA used alone showed a dramatic increase in iron content $(575 \mathrm{mg} / \mathrm{kg})$ and a decline $(390 \mathrm{mg} / \mathrm{kg})$ when both sources were combined.

Further study with different cultivars showed a significant interaction between cultivar and physical state of the medium to improve growth and multiplication rate in most cultivars tested. Comparison of four hazelnut cultivars viz., Geneva, C-16, Epsilon and Jefferson grown in semi-solid medium and TIS (Figs. 1e and 4a-h) showed that the plants in TIS had significantly greater shoot height (at least 2 fold), leaf area (at least 3 fold) and chlorophyll content in all cultivars tested. A significant interaction effect was observed between cultivar and physical state of the medium in promoting all parameters including number of shoots, number of nodes, shoot height, leaf area, chlorophyll a and chlorophyll b. All cultivars performed better for all growth parameters studied when grown in TIS compared to semi-solid medium. The number of shoots and multiplication rates were significantly increased in all cultivars except for Jefferson. However, the number of nodes was not significantly different between physical states of media or culture system in any of the cultivars tested. No significant difference in shoot height was observed among cultivars within each medium. C-16 (3.3 \pm 0.2$)$ produced a significantly greater number of shoots than Jefferson (2.1 \pm 0.4$)$ or Epsilon (2.2 \pm 0.2$)$ in TIS. However, among cultivars tested, there was no significant difference in number of shoots on semi-solid medium. Epsilon produced a significantly higher number of nodes than Geneva and Jefferson in semi-solid medium, although this difference was not observed in TIS. Among the four cultivars tested, C-16 had the highest multiplication rate on both semi-solid and TIS with a mean of $13.24 \pm 1.2$ and $28.4 \pm 2$, respectively. However, overall shoot multiplication rate increased in the TIS compared to semisolid medium. Geneva plantlets produced leaves with significantly higher leaf area. Mean chlorophyll a and b content were relatively higher in C-16 and Epsilon, however differences were not significant. Proliferated shoots rooted in the semisolid medium with $2.5 \mu \mathrm{M}$ IBA inducing the highest (83\%) response (Fig. 1f). Addition of FeEDDHA to semi-solid medium and the use of liquid medium showed no advantage, but reduced root induction and growth (data not shown). In vitro rooted plantlets acclimized well in a mist chamber and had a survival rate of $80 \%$ on transplanting to greenhouse (Fig. 1g). 


\section{Discussion}

Earlier studies on micropropagation of hazelnut indicated that the type of iron chelate in the growth medium was a critical factor in successful culture of hybrid hazelnut (Yu and Read 1995; Garrison et al. 2013). Our results of optimization of iron content in the culture medium confirmed that iron supplement as FeEDDHA or FeEDTA was essential for hazelnut multiplication and of the two, FeEDDHA was significantly more effective. FeEDDHA is more stable and less toxic at higher levels than $\mathrm{FeSO}_{4}$ or FeEDTA (Van der Salm 1994) and has increasingly become a preferred source of iron for micropropagation of various species (Nas and Read, 2001, Antonopoulou et al. 2007, Garrison et al. 2013). Combinations of iron supplements at moderate levels have been reported to improve shoot growth and chlorophyll content in rose (Van der Salm et al. 1994) and Carica papaya cultures (Castillo et al. 1997). In vivo application of FeEDDHA has also been reported to restore the green color in chlorotic leaves of soybean with a long-lasting effect in both soilless (Garcia-Marco et al. 2006) and field conditions (Schenkeveld et al. 2010).

A noteworthy observation, which contributed to the development of our micropropagation protocol was the efficiency of a combination of FeEDTA and FeEDDHA in stimulating higher proliferation of shoots. Both of these iron sources have been used for hazelnut cultures individually, but the effects of their combination have not been explored. This growth improvement may be due to enhanced iron availability leading to a higher iron content in the plantlets as indicated by iron uptake study (Fig. 3h). Enhanced shoot multiplication in our study may also reflect the influence of FeEDDHA on cytokinin metabolism; FeEDDHA has been shown to modulate cytokinin activity in Vaccinium genotypes (Shilbi et al. 1997). Interestingly, in our study the addition of FeEDDHA to rooting medium reduced root development suggesting cytokinin-like attribute of FeEDDHA. Optimum shoot and root development is known to be regulated by a balance of auxin:cytokinin in the tissue and its growth environment (Skoog and Miller, 1957). 
In many cases, micropropagation protocols are genotype specific and need to be tested on a range of cultivars. All four hazelnut cultivars tested in the present study produced healthy plantlets in TIS containing the medium supplemented with FeEDTA and FeEDDHA. The TIS had a significant beneficial effect on shoot development and multiplication rate as compared to the semi-solid medium. The use of TIS significantly increased the shoot height nearly by two fold, number of shoots by 1.5 -fold with over twice the multiplication rate and thrice the leaf area as compared to the semi-solid medium for all four cultivars with C-16 showing highest multiplication rate. Jefferson did not show a significant enhancement in multiplication rate, however, a significant increase in shoot height was observed. Variability in shoot multiplication responses has been observed earlier in hazelnut (Caboni et al. 2009), pear (Damiano et al. 2002) and rubber (Etienne et al. 1997). In general, TIS have been found to support mass multiplication of several species including Pyrus communis (Damiano et al., 2000), Curcuma longa (Adelberg and Cousins, 2006), Solanum tuberosum (Kamarainen-Karppinen et al., 2010) and grapevines (Harris and Mason, 1983). Growth promotion in TIS bioreactors is ascribed to a better availability and efficient uptake of nutrients, enhanced gas exchange with less build up of ethylene and toxic substances (Jackson 2002; Zobayed et al. 2003; Roels et al. 2005; Yang and Yeh 2008).

In conclusion, an efficient micropropagation protocol has been developed for hybrid hazelnut (Corylus spp.) cultivars by optimizing the source and concentration of iron supplements using TIS. Based on the multiplication rates routinely obtained, it is potentially feasible to raise approximately 300,000 plantlets of a responsive hazelnut cultivar per year starting with 100 established buds multiplied in 700 bioreactor vessels. The protocol utilizes buds from greenhouse or in vitro cultures and allows for continuous production all year around for commercial scale propagation. Additionally, the maintenance of stock plants by recycling of cultures and plants transplanted in the greenhouse can reduce the cost of maintaining source trees in the field. As such, these results can facilitate mass propagation of hazelnut cultivars and many other recalcitrant woody plant species of commercial interest. Plant response to micropropagation process is often genotype and cultivar specific and minor modifications in culture and growth milieus may be necessary for other species and cultivars. 


\section{Acknowledgements}

This research study was funded by the Ontario Centers of Excellence (OCE) and the Gosling Research Institute for Plant Preservation (GRIPP). We thank North American Plant Inc. (Oregon, USA) for providing the plant material.

\section{References}

Adelberg, J., and Cousins, M. 2006 Thin films of liquid media for heterotrophic growth and storage organ development: Turmeric (Curcuma longa) as a model plant. Hortscience 41(3): 539-542.

Antonopoulou, C., Dimassi, K., Therios, I., Chatzissavvidis, C., and Papadakis, I. 2007. The effect of FeEDDHA and of ascorbic acid on in vitro rooting of the peach rootstock GF-677 explants. Acta Physiol. Plant 29(6): 559-561.

Bassil, N., Mok, D., Mok, M., and Rebhuhn, B. 1992 Micropropagation of the hazelnut, Corylus avellana. Acta Hortic 300:137-140.

Caboni, E., Frattarelli, A., Giorgioni, M., Meneghini, M., and Damiano, C. 2009. Improving micropropagation of hazelnut Italian cultivars through temporary immersion system. VII international congress on hazelnut 845: 255-260.

Castillo, B., Smith, M., Madhavi, D., and Yadava, U. 1997. Interactions of irradiance level and iron chelate source during shoot tip culture of Carica papaya L. Hortscience 32(6): 1120-1123.

Damiano, C., Frattarelli, A., and Giorgioni, M. 2002. Micropropagation of pear through temporary immersion. Acta Hortic. 596:425-429.

Damiano, C., La Starza, S., Monticelli, S., Gentile, A., Caboni, E., and Frattarelli, A. 2005. Propagation of Prunus and Malus by temporary immersion. In Liquid Culture Systems for in vitro Plant Propagation, Edited by A.K. Hvoslef-Eide and W. Preil, Springer, Netherlands pp:243-251.

Driver, J.A., and Kuniyuki, A.H. 1984. In vitro propagation of paradox walnut rootstock. Hortscience 19(4): 507-509.

Etienne, H., Lartaud, M., Michaux-Ferrière, N., Carron, M., Berthouly, M., and Teisson, C. 1997. Improvement of somatic embryogenesis in Hevea brasiliensis (müll. arg.) using the temporary immersion technique. In Vitro Cell Dev. Biol.-Plant 33(2): 81-87.

Garrison, W., Dale, A., and Saxena, P.K. 2013. Improved shoot multiplication and development in hybrid hazelnut nodal cultures by ethylenediamine di-2-hydroxy-phenylacetic acid (Fe-EDDHA). Can. J. of Plant Sci. 93: 1-11.

Hand, C., Maki, S., and Reed, B.M. 2014. Modeling optimal mineral nutrition for hazelnut micropropagation. Plant Cell Tiss. Org. Cult. 119: 411-425. 
Hand, C., and Reed, B.M. 2014. Minor nutrients are critical for the improved growth of Corylus avellana shoot cultures. Plant Cell Tiss. Org. Cult. 119: 427-439.

Harris, R.E., and Mason, E.B. 1983. Two machines for in vitro propagation of plants in liquid media. Can. J. Plant Sci. 63(1): 311-316.

Hirata, S., Umezaki, Y., and Ikeda, M. 1986. Determination of Chromium( I I I), Titanium, Vanadium, Iron( I I I), and Aluminum by Inductively Coupled Plasma Atomic Emission Spectrometry with an OnLine Preconcentrating Ion-Exchange Column. Anal. Chem. 58: 2602-2606.

Jackson, M.B. 2002. Ventilation of plant tissue cultures. First International Symposium on Liquid Systems for In vitro Mass Propagation of Plants. Cost 843 Working Group, Norway pp 56-57.

Lichtenthaler, H.K., and Buschmann, C. 2001. Chlorophylls and carotenoids: Measurement and characterization by UV-VIS spectroscopy. Curr. Protoc. Food Analyt. Chem.: F4.3.1-F4.3.8.

Meguro, R., Asano, Y., Odagiri S, Li C., Iwatsuki, H., and Shoumura, K. 2007. Nonheme-iron histochemistry for light and electron microscopy: A historical, theoretical and technical review. Arch. Histol. Cytol. 70(1): 1-19.

Nas, M.N., and Read, P.E. 2001. Micropropagation of hybrid hazelnuts: Medium composition, physical state and iron source affect shoot morphogenesis, multiplication and explant vitality. Acta Hortic. 556: 251-258.

Nas, M.N., and Read, P.E. 2004. A hypothesis for the development of a defined tissue culture medium of higher plants and micropropagation of hazelnuts. Sci. Hortic. 101(1): 189-200.

Roels, S., Escalona, M., Cejas, I., Noceda, C., Rodriguez, R., Canal, M.J., Sandoval, J., and Debergh, P. 2005. Optimization of plantain (Musa AAB) micropropagation by temporary immersion system. Plant Cell Tiss. Org. Cult. 82: 57-66

Schenkeveld, WD.., Temminghoff, E.J., Reichwein, A.M., and van Riemsdijk, W.H. 2010. FeEDDHAfacilitated Fe uptake in relation to the behaviour of FeEDDHA components in the soil-plant system as a function of time and dosage. Plant Soil 332(1-2): 69-85.

Skoog, F., and Miller, CO. 1957 Chemical regulation of growth and organ formation in plant tissues cultured in vitro. In The Biological Action of Growth Substances, Symposia of the society for Experimental Biology No. 11. Edited by H.K. Porter. Cambridge University Press, Cambridge. pp. $118-131$.

Van der Salm Theo, P.M., Van der Toorn Caroline, J.G., Hänisch ten Cate Charlotte, H., Dubois, LAM, De Vries, D.P., and Dons, H.J.M. 1994. Importance of the iron chelate formula for micropropagation of Rosa hybrida L.'Moneyway'. Plant Cell Tiss. Org. Cult. 37(1): 73-77.

Yang, S-H., and Yeh, D-M. 2008. In vitro leaf anatomy, ex vitro photosynthetic behaviors and growth of Calathea orbifolia (Linden) Kennedy plants obtained from semi-solid medium and temporary immersion systems. Plant Cell Tiss. Org. Cult. 93:201-207.

Yu, X., and Reed, B.M. 1995. A micropropagation system for hazelnuts (Corylus species). Hortscience 30(1): 120-123. 
Zobayed, S.M.A., and Saxena, P.K. 2003. In vitro-grown roots: a superior explant for prolific shoot regeneration of St. John's wort (Hypericum perforatum L. cv 'New Stem') in a temporary immersion bioreactor. Plant Sci. 165 (3): 463-470.

\section{FIGURES}

Fig. 1 Micropropagation of hazelnut (Corylus sp.) (a) Liquid Lab ${ }^{\mathrm{TM}}$ Rocker temporary immersion bioreactor system, (b) shoot bud initiation, (c) shoot development on DKW basal medium after 4 weeks of culture, (d) shoot development from nodal explants after 4 weeks on semi-solid DKW medium containing 17.6 $\mu \mathrm{M}$ BA and $0.29 \mu \mathrm{M}$ GA3, $0.14 \mu \mathrm{M}$ IBA and $460 \mu \mathrm{M}$ FeEDDHA, (e) shoot proliferation from nodal explants after 8 weeks in TIS (f) in vitro raised rooted plantlets from semi-solid DKW medium containing $2.5 \mu \mathrm{M} \mathrm{IBA}$, and (g) in vitro raised hazelnut plants growing in the greenhouse.

Fig. 2 Effect of various concentrations of FeEDDHA alone or in combination with constant amount of $\mathrm{FeSO}_{4}(121.58 \mu \mathrm{M})$ on (a) shoot height, (b) number of shoots and (c) number of nodes of Geneva after six weeks of culture. Shoot explants failed to grow in the absence of FeEDDHA (control). Means from all the treatments were compared with each other. Columns with same letter indicate no significant difference $(\mathrm{P}<0.05)$.

Fig. 3 Iron detection in the leaf tissue of hazelnut plantlets grown at different concentrations of iron supplements (a) Control (CDKW), (b) $460 \mu \mathrm{M}$ FeEDDHA (CDKW), (c) $460 \mu \mathrm{M}$ FeEDDHA (MDKW), and (d) graphical representation of iron content in plantlets grown in medium containing different concentration of iron source provided by constant amount of $\mathrm{FeSO}_{4}(121.58 \mu \mathrm{M})+$ varying FeEDDHA (Blue line) and in plantlets grown in varying concentration of FeEDDHA (Red line). Arrows indicate iron pigments after staining; photographs were taken at same magnification level.

Fig. 4 Comparison of (a) Shoot height, (b) Number of shoots, (c) Number of nodes, (d) Multiplication rate, (e) Leaf area, (f) Chlorophyll a (g) Chlorophyll b and (h) Chlorophyll a/b of hazelnut cultivars (Epsilon, Jefferson, Geneva and C-16) in Liquid Lab ${ }^{\mathrm{TM}}$ Temporary Immersion system supplemented with $460 \mu \mathrm{M}$ of 
FeEDDHA in basal DKW medium after 8 weeks of culturing. Means from all the treatments were compared with each other. Columns with the same letter indicate no significant difference $(\mathrm{P}<0.05)$. 

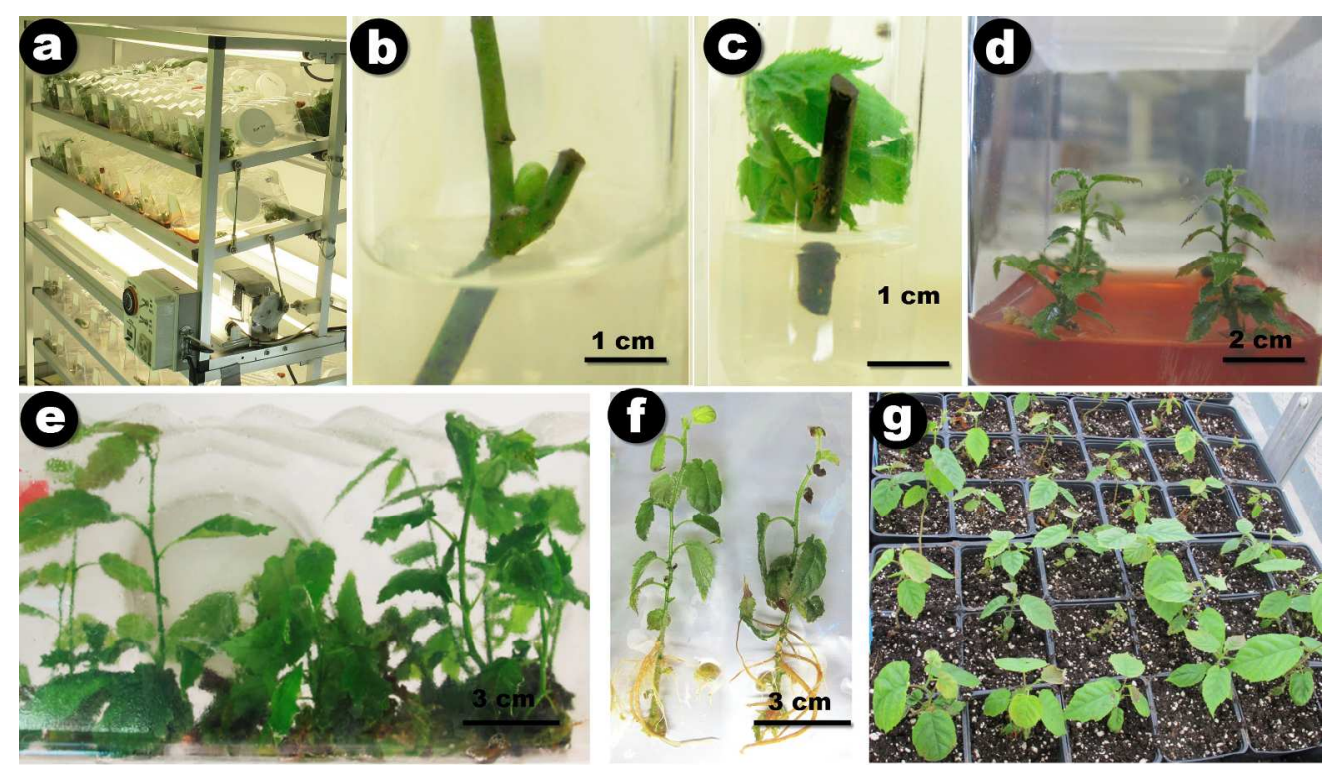

$220 \times 127 \mathrm{~mm}(300 \times 300$ DPI $)$ 

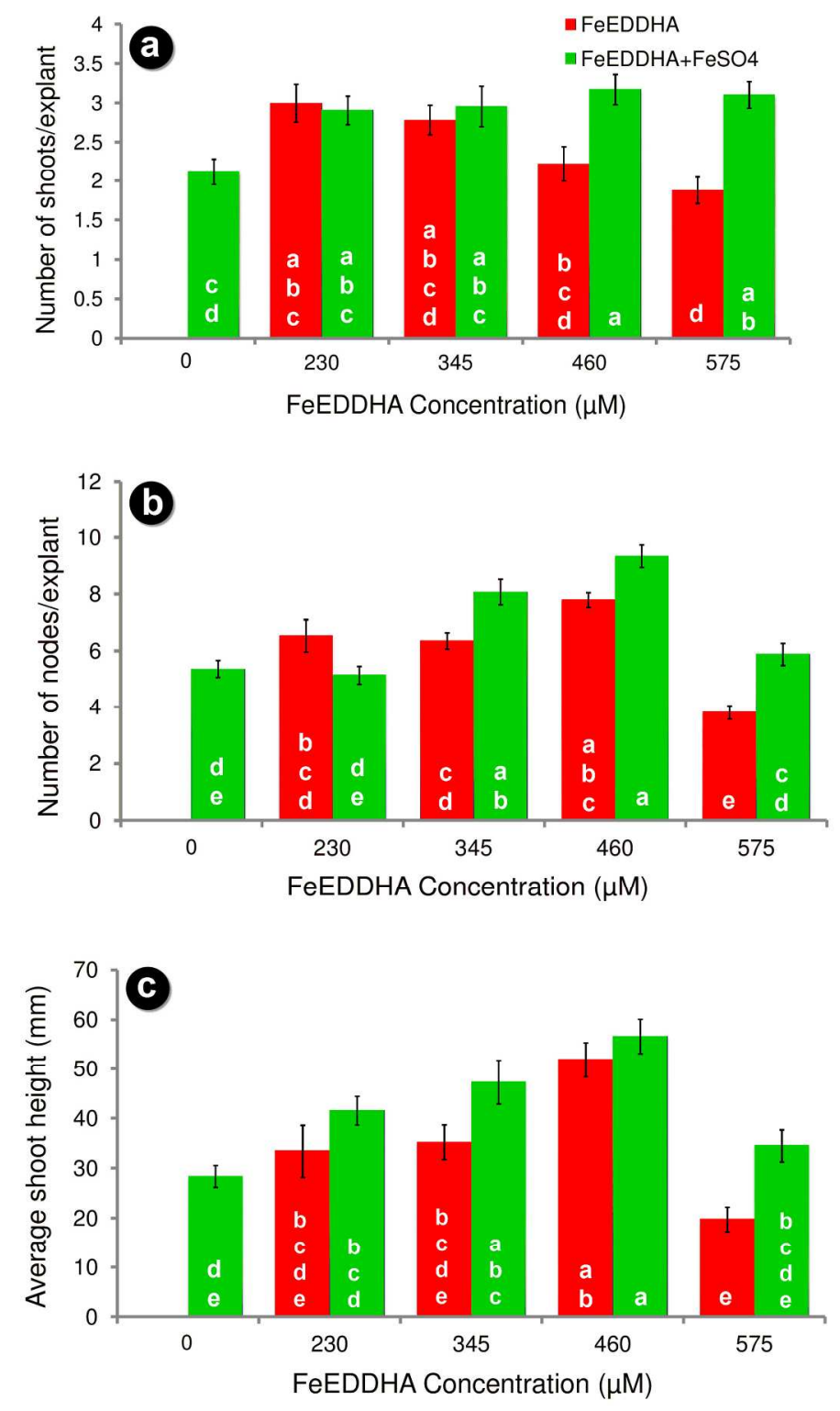

Figure 2

$157 \times 270 \mathrm{~mm}(300 \times 300$ DPI $)$ 

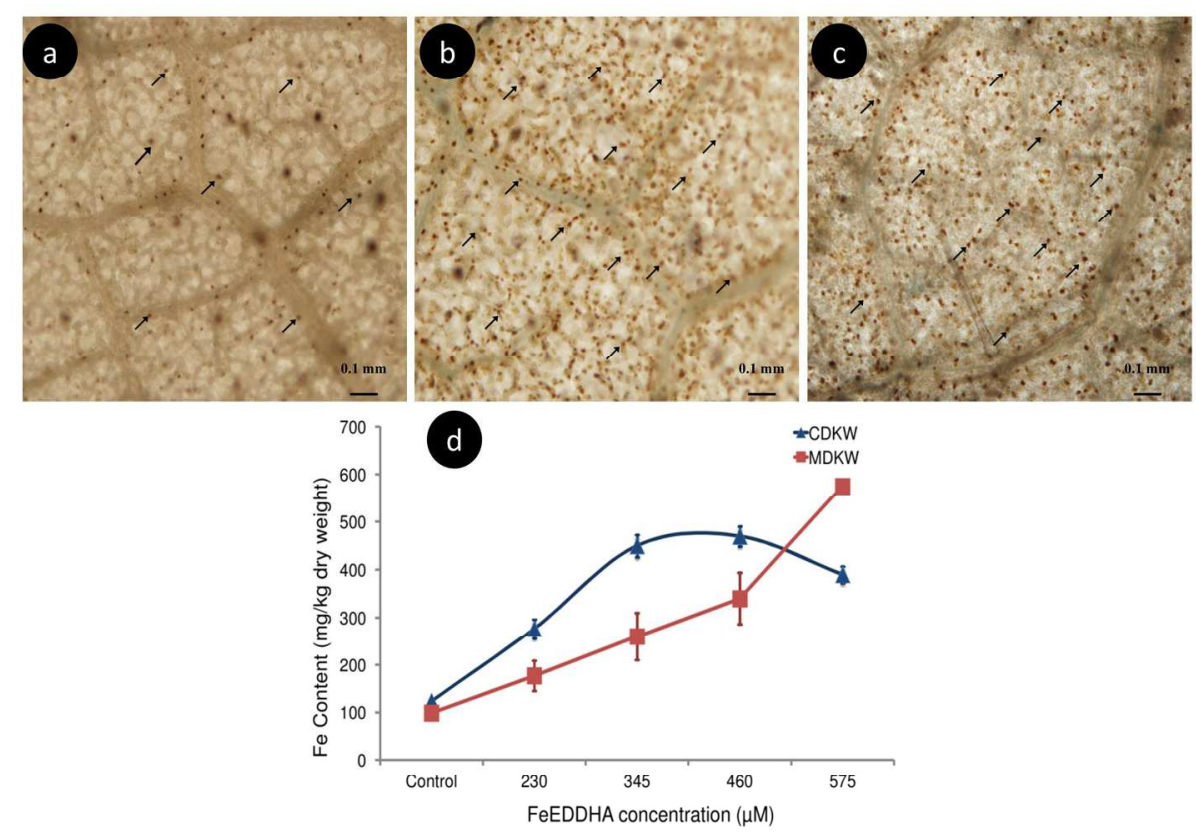

figure 3

$190 \times 142 \mathrm{~mm}(300 \times 300$ DPI $)$ 

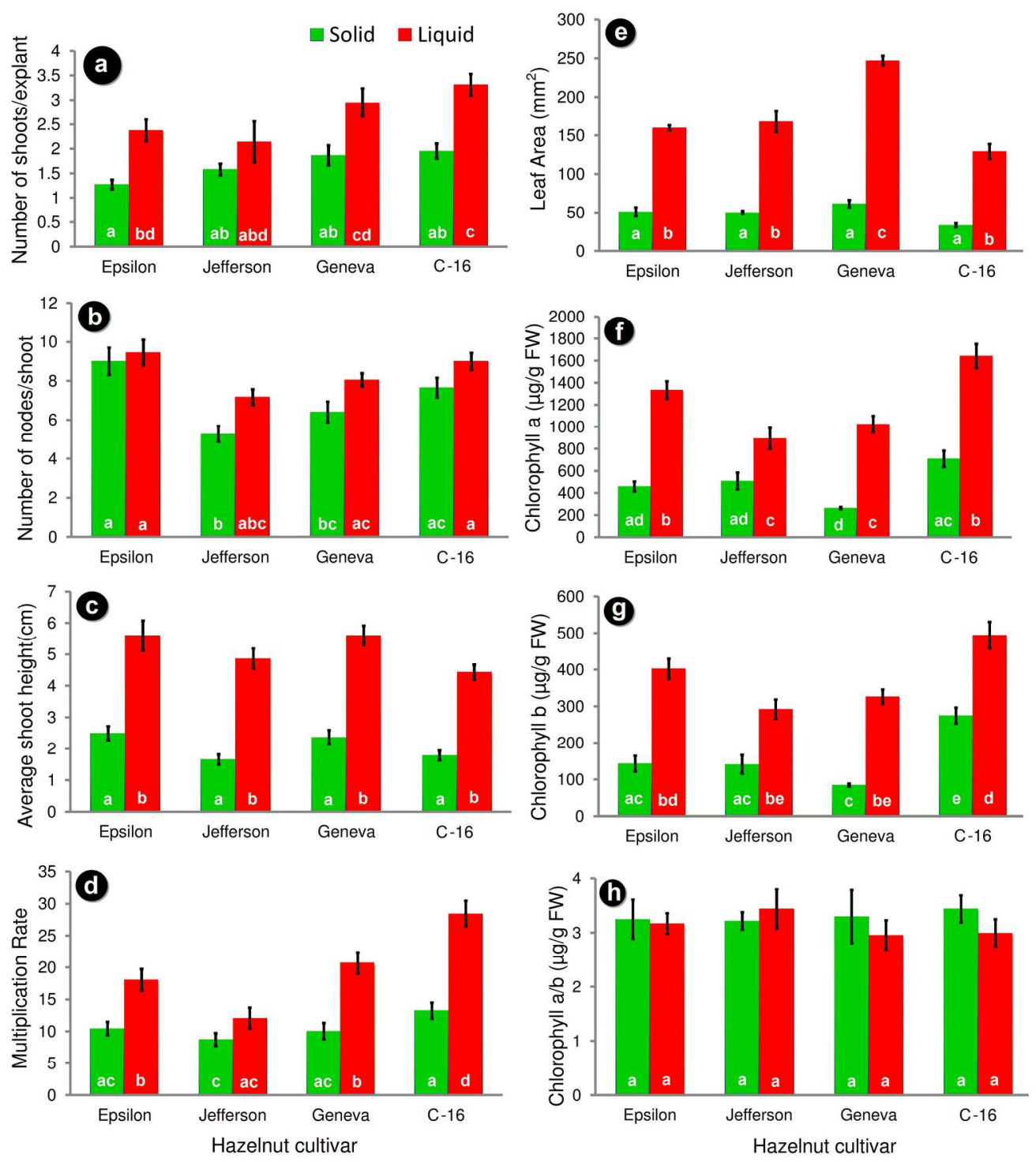

Figure 4

$190 \times 215 \mathrm{~mm}(300 \times 300$ DPI $)$ 